\title{
smORFer: a modular algorithm to detect small ORFs in prokaryotes
}

\author{
Alexander Bartholomäus ${ }^{\oplus 1,2, *}$, Baban Kolte ${ }^{2}$, Ayten Mustafayeva ${ }^{3,4}$, Ingrid Goebel ${ }^{2}$, \\ Stephan Fuchs ${ }^{5}$, Dirk Benndorf ${ }^{6,7}$, Susanne Engelmann ${ }^{\oplus 3,4}$ and Zoya Ignatova ${ }^{\oplus 2, *}$
}

${ }^{1}$ GFZ German Research Centre for Geosciences, Section Geomicrobiology, 14473 Potsdam, Germany, ${ }^{2}$ Inst. Biochemistry and Molecular Biology, Department of Chemistry, University of Hamburg, 20146 Hamburg, Germany, ${ }^{3} \mathrm{Helmholtz}$ Center for Infection Research, Microbial Proteomics, 38124 Braunschweig, Germany, ${ }^{4}$ Inst. Microbiology, TU Braunschweig, Braunschweig, Germany, ${ }^{5}$ Robert Koch Institute, Berlin, Germany, ${ }^{6}$ Otto von Guericke University, Bioprocess Engineering, 39106 Magdeburg, Germany and ${ }^{7}$ Max Planck Institute for Dynamics of Complex Technical Systems, Bioprocess Engineering, 39106 Magdeburg, Germany

Received December 07, 2020; Revised April 29, 2021; Editorial Decision May 13, 2021; Accepted May 18, 2021

\begin{abstract}
Emerging evidence places small proteins ( $\leq 50$ amino acids) more centrally in physiological processes. Yet, their functional identification and the systematic genome annotation of their cognate small openreading frames (smORFs) remains challenging both experimentally and computationally. Ribosome profiling or Ribo-Seq (that is a deep sequencing of ribosome-protected fragments) enables detecting of actively translated open-reading frames (ORFs) and empirical annotation of coding sequences (CDSs) using the in-register translation pattern that is characteristic for genuinely translating ribosomes. Multiple identifiers of ORFs that use the 3-nt periodicity in Ribo-Seq data sets have been successful in eukaryotic smORF annotation. They have difficulties evaluating prokaryotic genomes due to the unique architecture (e.g. polycistronic messages, overlapping ORFs, leaderless translation, non-canonical initiation etc.). Here, we present a new algorithm, smORFer, which performs with high accuracy in prokaryotic organisms in detecting putative smORFs. The unique feature of smORFer is that it uses an integrated approach and considers structural features of the genetic sequence along with in-frame translation and uses Fourier transform to convert these parameters into a measurable score to faithfully select smORFs. The algorithm is executed in a modular way, and dependent on the data available for a particular organism, different modules can be selected for smORF search.
\end{abstract}

\section{INTRODUCTION}

Next-generation sequencing (NGS) technologies enable a rapid and easy detection of genomic information of new species. However, delineating protein-coding open reading frames (ORFs) in genomes after sequencing and de novo genome assembly remains still a challenge. After the pioneering effort of Fickett to unify concepts on how to define protein-coding sequences (1), further criteria have been added to increase the confidence in de novo identifications. These include intrinsic signals involved in gene specifications (e.g. start and stop codon, splice sites), conservation patterns in related genomes with weighted conservation depending on evolutionary distance and verification with known ORFs or protein sequences $(2,3)$. Classically, these rules in the genome annotation protocols are performing well only on larger ORFs which span at least 100 codons $(4,5)$, thus small ORFs (smORFs) shorter than 100 codons are systematically underrepresented and cannot be identified by common algorithms (6). Mounting evidence suggests crucial functions for smORFs in cellular and molecular processes in both eukaryotes (6-13) and prokaryotes (14-22). However, systematic identification of functional small proteins or microproteins (also called micropeptides) remains challenging both experimentally and computationally.

Recent developments of NGS technologies to probe the position of translating ribosomes with codon precision ribosome profiling or Ribo-Seq (23), enable detecting actively translated ORFs by capturing ribosome-protected fragments (RPFs) and is used to empirically annotate coding sequences (CDSs). Several new previously unannotated ORFs, including smORFs, have been identified mostly in eukaryotes (8,24-26). Some studies oppose that RPFs alone are sufficient to classify a transcript as protein-coding or non-coding (27). Alternatively, Poly-Ribo-Seq which specif-

\footnotetext{
"To whom correspondence should be addressed. Tel: +49 4042838 2332; Email: zoya.ignatova@chemie.uni-hamburg.de Correspondence may also be addressed to Alexander Bartholomäus. Email: bartholomaeus.alexander@gmail.com

(C) The Author(s) 2021. Published by Oxford University Press on behalf of Nucleic Acids Research.

This is an Open Access article distributed under the terms of the Creative Commons Attribution License (http://creativecommons.org/licenses/by/4.0/), which permits unrestricted reuse, distribution, and reproduction in any medium, provided the original work is properly cited.
} 
ically sequences polyribosomes separated through sucrose gradients is suggested as more stringent approach in isolating translated ORFs (28). mRNAs translated by more than one ribosome (i.e. polyribosomes) are classically defined as genuinely translated mRNAs. However, studies in eukaryotes show that monosomes, initially considered as nontranslating ribosomes, are in fact elongating ribosomes involved in translation of low-abundance transcripts or such with much slower initiation than elongation (29), or bear tissue-specific translation signature (30). Moreover, given that a ribosome protects on average 26-30 nt, this approach may miss a significant fraction of expressed transcripts and in particular very short smORFs (less than 10 amino acids) whose size might permit translation by a single ribosome, and thus, they migrate in the monosomal fraction. RiboSeq combined with an antibiotic treatment that specifically stalls ribosomes at translation initiation site (TIS-RiboSeq) selects for potential new initiation sites and allows detecting new ORFs in non-coding regions or overlapping ORFs which overlap with annotated ORFs and are undistinguishable in the Ribo-Seq data sets $(8,19,22,25,31-34)$.

Complementing Ribo-seq with computational predictions revealed several hundred smORFs in eukaryotes $(8,24,26,35,36)$. The crucial metrics they use, is the enrichment of RPFs in ORFs and the 3-nt periodicity characteristic for genuinely translating ribosomes. These approaches have difficulties evaluating prokaryotic genomes due to their unique architecture, including polycistronic messages, large fraction of overlapping ORFs, leaderless translation and lack of classical ribosome-binding site (i.e. with direct start of translation from the start codon $(37,38)$ ). The resolution of the prokaryotic Ribo-Seq data is lower than that in eukaryotes due to the intrinsic properties of the nucleases used in prokaryotic Ribo-Seq experiments (39), which often results in imperfect periodicity. Together, this makes a genome-wide identification of smORFs encoding functional small proteins in prokaryotes even more challenging.

Here, we present a new algorithm, smORFer, for identifying smORFs by integrating genomic information, structural features, Ribo-Seq and TIS-Ribo-Seq to faithfully select translated and initiated ORFs, respectively. The algorithm is executed in a modular fashion and various modules can be selected dependent on the data availability for each organism. smORFer is versatile and suitable for every organism, but shows high confidence of predictions for in particularly difficult-to-annotate smORFs in bacteria.

\section{MATERIALS AND METHODS}

\section{Data sets used in the analysis}

We generated two biological Ribo-Seq replicates for Staphylococcus aureus Newman and downloaded Escherichia coli MG1655 (Ribo-Seq, GSM3455899 and retapamulintreated TIS-Ribo-Seq, GSM3455900 (19)) and Bacillus subtilis data (Ribo-Seq, GSM872395 and GSM872397, (40)) from the Gene Expression Omnibus (GEO) repository. The Ribo-Seq data for $S$. aureus Newman were uploaded in GEO under accession number GSE150601. Mass spectrometry data for $S$. aureus are from (41) and for E. coli from (42).

\section{Ribo-Seq of $S$. aureus}

Cells grown in TSB medium (pancreatic digest of casein $17 \mathrm{~g} / 1$, enzymatic digest of soya bean $3 \mathrm{~g} / 1, \mathrm{NaCl} 5 \mathrm{~g} / 1$, $\mathrm{K}_{2} \mathrm{HPO}_{4} 2.5 \mathrm{~g} / 1$, glucose $2.5 \mathrm{~g} / 1, \mathrm{pH} 7.3$ ) to $\mathrm{OD}_{550}=1$ were harvested by rapid centrifugation, resuspended in ice-cold $20 \mathrm{mM}$ Tris lysis buffer $\mathrm{pH} 8.0$, containing $10 \mathrm{mM} \mathrm{MgCl}_{2} \cdot 6$ $\mathrm{H}_{2} \mathrm{O}, 100 \mathrm{mM} \mathrm{NH} \mathrm{H}_{4} \mathrm{Cl}, 0.4 \%$ Triton-X-100, 4 U DNase, 0.4 $\mu 1$ Superase-In (Ambion), $1 \mathrm{mM}$ chloramphenicol and disrupted by homogenisation (FastPrep-24 ${ }^{\mathrm{TM}}$, MP Biomedicals) with $0.5 \mathrm{ml}$ glass beads (diameter $0.1 \mathrm{~mm}$ ). $100 \mathrm{~A}_{260}$ units of ribosome-bound mRNA fraction were subjected to nucleolytic digestion with 10 units/ $\mu 1$ micrococcal nuclease (Thermofisher) in buffer with $\mathrm{pH} 9.2(10 \mathrm{mM}$ Tris pH 11 containing $50 \mathrm{mM} \mathrm{NH}_{4} \mathrm{Cl}, 10 \mathrm{mM} \mathrm{MgCl} 2,0.2 \%$ triton $\mathrm{X}-100,100 \mu \mathrm{g} / \mathrm{ml}$ chloramphenicol and $20 \mathrm{mM} \mathrm{CaCl}_{2}$ ). The rRNA fragments were depleted using the $S$. aureus riboPOOL rRNA oligo set (siTOOLs, Germany) and the library preparation was performed as previously described (43).

\section{Data processing and mapping}

Raw sequencing reads were trimmed using FASTX Toolkit (quality threshold: 20) and adapters were cut using cutadapt (minimal overlap of $1 \mathrm{nt}$ ). The following genome versions were used for mapping: E. coli U00096.3, S. aureus NC_009641.1 and B. subtilis NC_000964.3. Genomes and annotations were downloaded from NCBI (January 2020). In the first step of mapping, reads mapping to rRNAs were discarded. Thereafter, reads were uniquely mapped to the reference genomes using Bowtie (44), parameter settings: -1 16 -n 1 -e 50 -m 1 -strata -best. Non-uniquely mapped reads were discarded. The total number of mapped reads are summarized in Supplementary Table S1.

The peptide identification for $E$. coli was performed using the dataset PXD000498 (mascot_daemon_merge.mgf) (42) available at PRIDE (45). To identify a MASCOT (version 2.6) (46) search against the smORF candidates (taking only longest smORF for candidates sharing the same stop codon) and all protein coding genes $(4,243$ sequences) and the respective decoy database was carried out with search parameters as previously published (42).

\section{smORFer workflow}

The workflow of smORFer, which is executed in a modular way, is summarized in Figure 1. Several simple counting and filtering steps are performed using BEDTools (47), e.g. ORFs in non-annotated regions where filter by intersectBed and counting read was done using coverageBed. The first part of Module $\mathrm{A}$ is required to define the boundaries of all putative ORFs. The selection is further refined by the structural properties that are intrinsic to protein-coding sequences. Modules B and C add further confidence to the detected smORF candidates and can be executed either independently or together; the latter increases the detection of true positive novel smORFs.

Genome-based ORF detection (Module A). A list of putative ORFs was generated using modified Perl script 

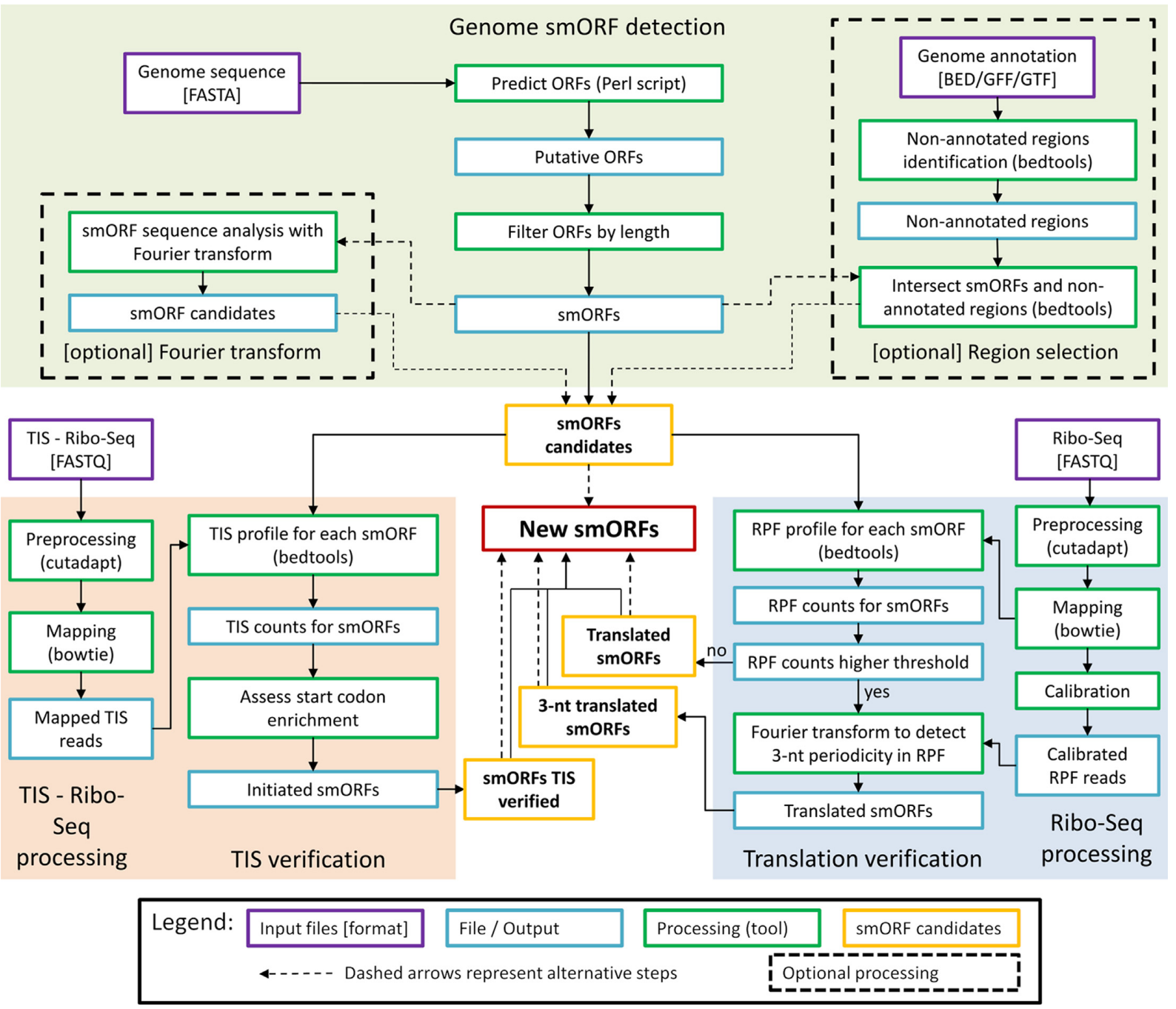

Figure 1. General scheme of smORFer algorithm with its three modules that evaluate genomic information (module A, green), translation and 3-nt periodicity in the RPFs from Ribo-Seq data (module B, blue), and TIS from TIS-Ribo-Seq (module C, orange).

(48); it generates putative ORFs with in-frame start and stop codon. We used four start codons, ATG, GTG, TTG, CTG, which are the most common in prokaryotes (49), and the three uniform stop codons, TGA, TAG, TAA. smORFer separated smORFs based on their location, e.g. in the non-annotated and annotated regions, and also contains a strand-specific filter for selecting the region.

To detect whether a putative smORF potentially encodes peptides or proteins, i.e. exhibits 3-nt sequence periodicity of the CDS, and hence, would be potentially translated, we used Fourier transform (FT; implemented as R's base fft function) of the GC content of each single gene, i.e. for each single ORF this is a vector of 0 's and 1's. The signal is first normalized to the ORF length as the signal intensity depends on the ORF length. In this 3-nt periodic pattern the 1.5 -nt period is always present along with the 3 -nt period regardless of the length of the putative ORF. Thereafter, we build the fraction of normalized signal at the period of $3 \mathrm{nt}$ and divide it by the arithmetic mean of the signal between both $3 \mathrm{nt}$ and $1.5 \mathrm{nt}$ periods.
Detection of translated ORFs from Ribo-Seq data including read processing (Module B). Ribo-Seq data are first mapped and smORFs with a minimum of five RPFs are selected and assigned as 'translated'. A coverage of $\geq 5 \mathrm{RPF}$ counts is on average above the counting error for short ORFs in Ribo-Seq data sets $(23,50)$ and we suggest it to be used as an arbitrarily cutoff when biological replicates are not available. Otherwise, the reliable minimum read counts per gene should be determined individually for each RiboSeq using variability analysis of the counting statistics of two independent biological replicates that also assesses the influence of counting noise $(23,43)$.

The calibration procedure assigns for each RPF the codon at the ribosomal $\mathrm{A}$ or $\mathrm{P}$ site, allowing for tracking the codon-wise periodic pace of ribosomes along ORFs. To position a read at the ribosomal $\mathrm{A}$ or $\mathrm{P}$ site, the reads are first binned by length and the offset is determined for each read length bin individually as described ((51); all scripts are available here: https://github.com/ AlexanderBartholomaeus/MiMB_ribosome_profiling). For prokaryotic Ribo-Seq data sets, a calibration using 3' ends, 
i.e. to the termination codons, is recommended since the nucleases used to generate RPFs in bacteria cleave in a sequence selective manner with somewhat less variations at the $3^{\prime}$ ends (52). The read length distributions vary between data sets likely because of different experimental protocols (53) and at least four to five highest read length bins should be considered. Here, we considered for E. coli and B. subtilis read length bins of 27-30 nt and for $S$. aureus 24-28 nt bins with an offset for the A site of $11 \mathrm{nt}$ for 24-28 nt and $12 \mathrm{nt}$ for 29-30 nt. Alternatively, other algorithms that extract A or $\mathrm{P}$ site from the RPF reads can be used. Similarly to our approach, Plastid (54) and RiboProfling (55) compute the P site by stratifying the reads in bins according to their length and treating each bin independently yield variable offsets across bins. riboWaltz (56), a two-step $\mathrm{R}$ algorithm, computes the $\mathrm{P}$ site with a high accuracy using a coherent single offset.

Calibration requires good read coverage, hence smORFs with a coverage of $100 \mathrm{RPFs}$ per kilobase of ORF length (RPK) were further subjected to FT analysis to determine the 3-nt or codon periodicity of the calibrated RPF profile. Usually, a coverage of 100 RPK (i.e. 1 read per $10 \mathrm{nt}$ ) results in a good FT analysis. smORFs with a 3-nt periodicity in the RPF coverage are classified as '3-nt translated'. Next, the 3-nt or codon periodicity of the calibrated RPF profile is subjected to FT and a score is extracted from the mean of the signal between the periods of $3 \mathrm{nt}$ and $1.5 \mathrm{nt}$. The threshold $(\mathrm{FT}>2)$ is determined from the cumulative distributions of $F T$ values for 2,315 protein-coding ORFs with $\geq 100$ RPK. smORFs with a FT value higher of 2 are classified then as '3nt-translated'. smORFs with low RPF coverage, for which a 3-nt periodic signal in the RPF profiles could not be determined, are sorted as 'translated'. Note, that 'translated' smORFs should be also kept as they could be true hits, but their relatively low translation levels, with only few RPFs, preclude calibration and FT analysis.

Detection of TIS (Module C). Ribo-Seq is performed in the presence of translation initiation inhibitor; here, for E. coli retapamulin was used (19). TIS-Ribo-Seq was processed in the same way as Ribo-Seq. The middle nucleotide of each RPF is extracted and used in further analysis; in the case of even read length, the $3^{\prime}$ nucleotide of the first half of an RPF is taken (51). It should be noted that reads from TIS-Ribo-Seq cannot be calibrated, because of the skewed coverage at initiation and the lack of coverage at termination; the latter prevents calibration at both start and stop codons (51). Moreover, a manual assignment of the offset is not possible, because retapamulin binds to the peptidyltransferase center in both presence and absence of initiator fMet-tRNA $(19,57,58)$, thus blurring the P-site assignment over at least two codon positions. For each smORF, the middle-nucleotide TIS counts over the three nucleotides of the start codon and one codon upstream and downstream of the start are summed up and ORFs with more than 5 RPFs are classified as having true TIS.

\section{Operating system and $\mathbf{R}$ versions, scripts and examples}

We used Ubuntu 18.04 LTS as the operating system. For data analysis and visualization, we used $\mathrm{R}$ (3.5.0) includ- ing packages seqinr (3.6-1) and Biostrings (2.50.2) which are available on all operating system. Scripts, example calls and files (except BAM files because of their large size) for smORFer using $E$. coli data sets are available at https:// github.com/AlexanderBartholomaeus/smORFer.

\section{RESULTS AND DISCUSSION}

\section{Design of the smORFer: a modular algorithm to detect smORFs}

The availability of various sequencing data (DNASeq, Ribo-Seq, TIS-Ribo-Seq) for different organisms may largely vary, hence we sought to develop an algorithm - smORFer - with a modular design which uses various data sets to detect putative smORFs. smORFer combines three modules which utilize different inputs and can be used independently or in combination to increase the confidence in smORFs annotation (Figure 1). The three inputs are: (i) the genomic nucleotide sequence for module A 'Genome-based smORF detection', (ii) Ribo-Seq data for module B 'Detection of translated ORFS' and (iii) TIS-Ribo-Seq for module C 'Detection of TIS' (Figure 1).

Genome-based ORF detection. This module uses genomic data as an input to first predict putative ORFs in a lengthindependent manner. In all three organisms tested, we detected a large number of putative smORFs with a length between 3 and 50 codons (including the stop codon). We restricted the maximal length cutoff to $\leq 50$ codons; the length of 50 codons has been defined for the category of small or micropeptides $(22,59)$. The algorithm, however, can perform calls for ORFs at any length. A single amino acid ORF, although theoretically possible to be produced from a start-stop-ORF (19), does not fulfil the criteria for a peptide and was not considered. We used 3 codons (i.e. including start and stop codons) as it will encode the shortest peptide, i.e. a dipeptide. We used four start codons, ATG, GTG, TTG, CTG, which are the most common in prokaryotes (49), and the three uniform stop codons, TGA, TAG, TAA.

Analysis of the genomes from the three organisms revealed a well-defined 3-nt sequence periodicity within the genomic DNA sequences of the CDSs (Figure 2), which is a characteristic feature of protein-coding CDSs (60). Next, we included a step to assess the discrete genomic 3nt sequence periodicity of each putative ORF using Fourier transform (Figure 3A, B, Supplementary Figure S1). To decrease the number of false-positives, we used a relatively restrictive cutoff (FT > 3, Figure 3C) which detects appr. 70\% of known ORFs. For comparison, a cutoff of 2 would detect $85 \%$ of the known ORFs.

Detection of translated ORFs from Ribo-Seq data. This module assesses the translation of each ORF from a RiboSeq data set (Figure 1). First, to filter out ORFs with a translation level below the threshold of sporadic expression, smORFer selects ORFs with a minimal coverage ( $\geq 5 \mathrm{RPFs}$ ) and categorizes them as translated. This threshold, inferred from earlier data sets $(23,50)$ provides a good balance of true false-positives and false-negatives as revealed by the comparison with experimentally verified smORFs (Figure 


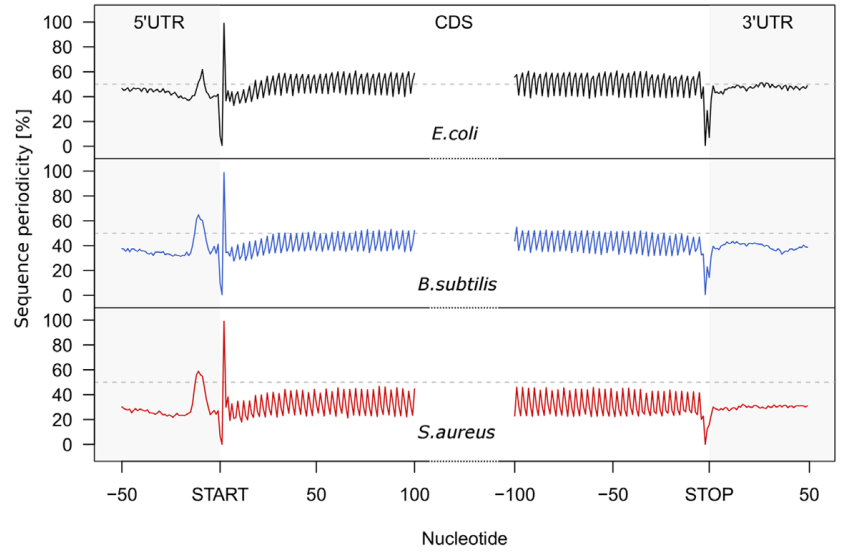

Figure 2. Metagene analysis of the genomic sequence periodicity across the $5^{\prime}$ UTRs, CDSs and $3^{\prime}$ UTRs of all protein-coding transcripts in E. coli (black), B. subtilis (blue) and S. aureus (red). ORFs are aligned at the start or stop codon, respectively. Note that the GC content differs among organisms and is $51 \%$ for E. coli, $44 \%$ for B. subtilis and $33 \%$ for S. aureus. Only non-overlapping protein-coding ORFs are considered. The horizonal dashed line denotes the average structure of a hypothetical genome with $50 \% \mathrm{GC}$ content.

4A). Genuinely translated ORFs exhibit a 3-nt periodicity in their RPF coverage, hence at a second stage, ORFs undergo a 3-nt periodicity analysis which is assessed again using FT (Figure 4B, C). smORFs over the threshold (FT $>2$, Figure 4D) are categorized as 3-nt translated. In the FT analysis of the calibrated RPFs we used a restrictive cutoff (FT $>2$, Figure 4D) which detects 512 of the known protein-coding ORFs in E. coli. Usually the 3-nt pattern is well detectable in smORFs with a good coverage, yet we do not discard smORFs with no discernible periodic RPF coverage (translated category) as they could be still expressed but translated at low level.

Detection of TIS. This module uses as input TIS-RiboSeq data. To block the ribosomal transition from initiation into elongation and to detect bona fide initiating ribosomes in prokaryotes, several antibiotics have been used so far, e.g. retapamulin (19), Onc112 (22), tetracycline (33). Thereby, retapamulin shows the sharpest peak at initiation. Considering the middle nucleotide of each TIS-Seq read and summing up TIS counts at the start codon including one codon upstream and downstream (Supplementary Figure S2), smORFer selects smORFs with $\geq 5$ RPFs at TIS (the same cutoff as for translated above) and categorizes them as translated with TIS signal (Figure 1, Table 1).

\section{Performance of smORFer for de novo identification of SmORFs}

Here, we employed the smORFer in predicting smORFs in three different organisms, E. coli, B. subtilis and S. aureus. For all three organisms Ribo-Seq data are available, and TIS-Ribo-Seq only for $E$. coli. In all three microorganisms tested, based on the genomic sequence and using the first search criterion, we detected a large number of putative smORFs with a length between 3 and 50 codons (>300 000, Table 1). Selection by this simple feature (48) revealed a large portion of overlapping smORFs, i.e. smORFs with different start codons but terminated by the same stop codon. Four different start codons, ATG, GTG, TTG and CTG, which are the four most used in bacteria, were used as selection criterion. Thereby, in E. coli their usage differs by several orders of magnitude, e.g. the usage is ATG $81.8 \%$, GTG $13.8 \%$, TTG $4.34 \%$ and CTG $0.024 \%$ (49). This start codon usage has been deduced from annotated (large) ORFs, but since smORFs may follow non-canonical codons and rules of initiation $(37,38)$, we kept all four codons with equal weight in the search. This initial step is required to set the boundaries of all possible putative ORFs.

Compared to the $5^{\prime}$ and $3^{\prime}$ UTRs, the coding sequences (CDS) of all three organisms exhibit a well-defined 3-nt sequence periodicity which is independent of the GC content of the organism (Figure 2). Even S. aureus genome with the lowest GC content (33\% GC content) shares the same 3- $n t$ periodic sequence pattern. Thus, we reasoned that smORFs, if protein or peptide coding, would share the same 3-nt sequence periodicity like annotated long CDSs encoding large proteins. To extract the 3-nt sequence periodicity of the genomic sequence, we subjected all smORFs to FT analysis which converts this characteristic pattern into a score (Figure 3 and Supplementary Figure S1) and used it as a further filtering criterion in module A (Table 1). Because of quite short smORF length and lower signal-to-noise ratios (61), we used a stringent FT score to select smORFs potentially encoding peptides (FT > 3, Figure 3C). Even with this stringent criterion, although it significantly reduced the number of potential candidates, the number of smORFs remained relatively large (Table 1).

Again, the majority of the detected smORFs in this step remained the overlapping ones, i.e. with distinct start codons but terminated by the same stop codon. Within these, the distribution of smORFs initiated with ATG, GTG, TTG and CTG was for E. coli $900,625,815$ and 739 , for B. subtilis 702, 422, 609 and 372, and for $S$. aureus 2356 , 1212, 2227 and 800, respectively. Notably, the distribution among the start codons in the putative smORFs is relatively balanced between these four start codons unlike their skewed distribution in initiating long annotated ORFs (49). At this stage, in order to not miss non-canonically initiated smORFs, we do not apply further selection criteria.

Next, using Ribo-Seq data we analyzed the translation status of the smORFs with 3-nt sequence periodic pattern (module B, Figure 1). In total, 3079, 6595 and 2105 smORFs for E. coli, S. aureus and B. subtilis, respectively, were selected with RPFs over the threshold (named translated candidates, Table 1). Overall, the identified smORFs were translated at very low level, exhibiting only few RPFs. Next, we applied a more stringent criterion for selecting genuinely translated ORFs and assessed the 3-nt periodicity of their RPF profile which is a characteristic feature of a genuine translation. For this, the RPFs were precisely positioned within ORFs, or calibrated by aligning their $3^{\prime}$ ends to the stop codons (52) - a key step in obtaining a codon resolution and extract 3-nt periodicity of the RPF profile. The calibrated RPFs to the ribosomal A site were then subjected to FT analysis which converts this 3-nt characteristic pattern of the RPF coverage into a score. SmORFs with FT $\geq 2$ were defined as 3 - $n$ t translated candidates (Table 1 ). In this step, 175, 555 and 168 non-annotated smORFs were 

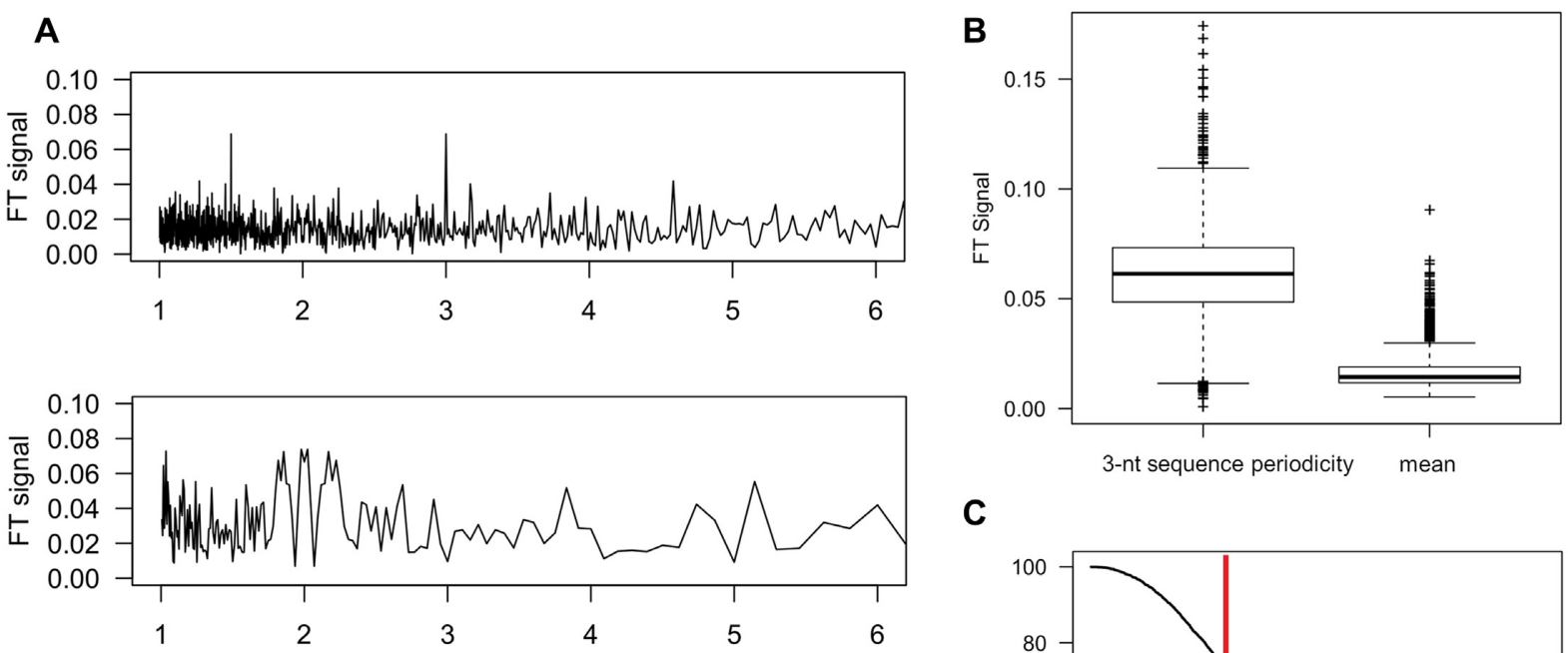

C
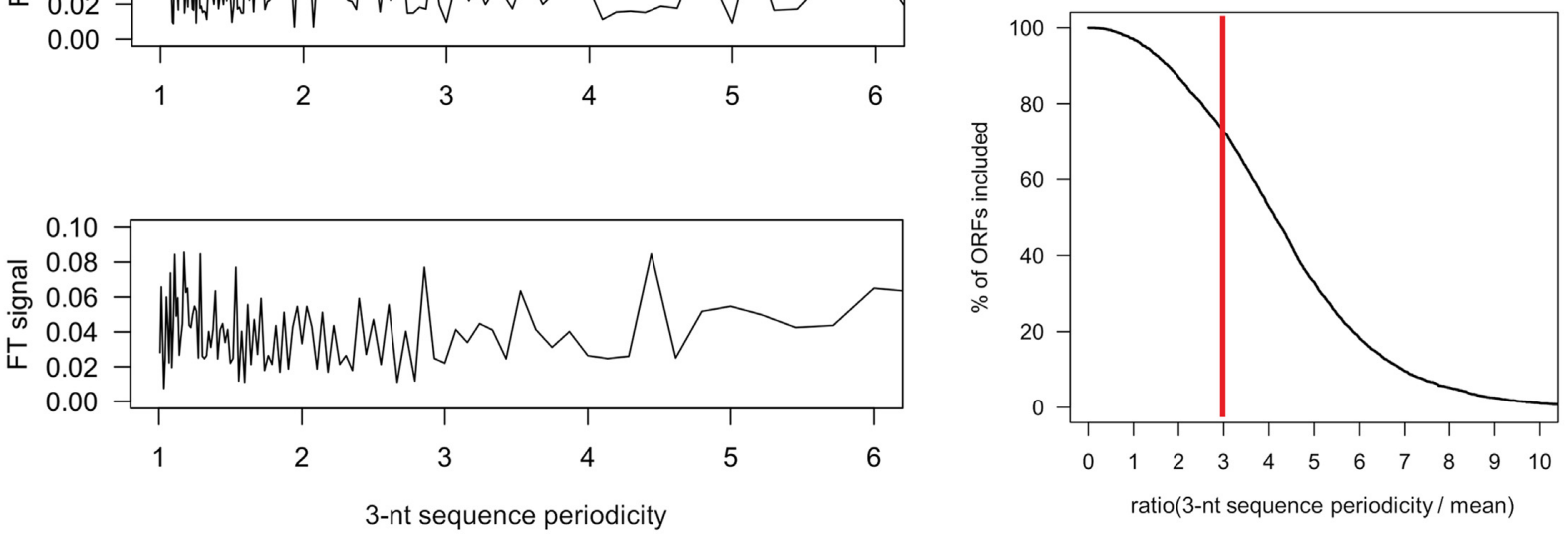

Figure 3. Fourier transform (FT) of the genomic 3-nt sequence periodicity in ORFs. (A) Averaged 3-nt sequence periodicity of protein-coding ORFs (upper panel), intergenic region (middle panel) and non-protein coding gene (e.g. 5S rRNA, lower panel). (B) The FT signal normalized by the ORF length at 3-nt sequence periodicity (left) and by the arithmetic mean of the signal between periodicity at 1.5 and 3 (right) for protein-coding ORFs. (C) Cumulative distributions of FT values (from B) for protein-coding ORFs. Vertical red line, cutoff of 3.

discovered in E. coli, S. aureus and B. subtilis, respectively (Table 1).

For E. coli, a TIS-Ribo-Seq data set using retapamulin to stall imitating ribosomes was available (19), which we used for further verification of both translated and 3-nt translated categories in module B (Table 1). Inspection of the TIS coverage in the annotated protein-coding ORFs showed that retapamulin crisply stalls over the start codon including one codon upstream and downstream of it, with a maximum coverage centered over the start codon (Supplementary Figure S2). From the 3079 translated smORFs, 160 possessed a TIS signal and from the 1753 -nt translated 16 (Table 1). The marked reduction of the number of potential candidates from these selected in the translated category emphasizes the importance of using various data sets to enhance stringency and confidence in smORFs identification and select genuinely translated candidates from translational noise.

In $E$. coli, using all three modules the algorithm successfully detected the experimentally verified smORFs, including also some recently identified smORFs with manually assessed TIS $(19,22)$ (Figure 5A and Supplementary Figure S3). Notably, the number of detected known smORFs by both, RPF and TIS signal, increased linearly with the RPF counts of smORFs (Figure 4A), suggesting an expressiondependent effect. smORFer detected new smORFs (Fig- ure 5B), many of which were overlapping and counted as independent in the modules A and B (Table 1). The true power of TIS-Ribo-Seq is in the precise positioning of the likely true start codon and thus, selecting true translated smORFs from overlapping frames (Figure 5C). Since TIS and RPF data are strand specific, we can clearly distinguish signals from each DNA strand, thus, precisely assigning smORFs ( $y i b X, y i b X-S, y i b H)$ on the opposite strand to the waaL ORF (Figure 5C). smORFer is also able to unambiguously assign overlapping ORFs on the same strand (Figure 5C), given that the TIS signals are separated by minimum $3 \mathrm{nt}$ (Supplementary Figure S2). The algorithm may miss some cases of completely overlapping ORFs, initiated through adjacent start codons $(<3 n t$, Figure 5B), although long stretches of overlap among ORFs are fairly rare (Supplementary Figure S4). It is worth mentioning, that retapamulin is so far the only initiation inhibitor for Gram-negative bacteria, that exhibits such precise inhibition at start codons (19) and allows for exact detection of TIS. Other antibiotics show much broader coverage across initiation sites and are not always precisely centered at the initiation codon $(22,33)$. To decrease the falsepositive hits, in particular for very short smORFs, we recommend executing restrictive call with a coverage over the start codon and expand it by maximally one codon at each side. 

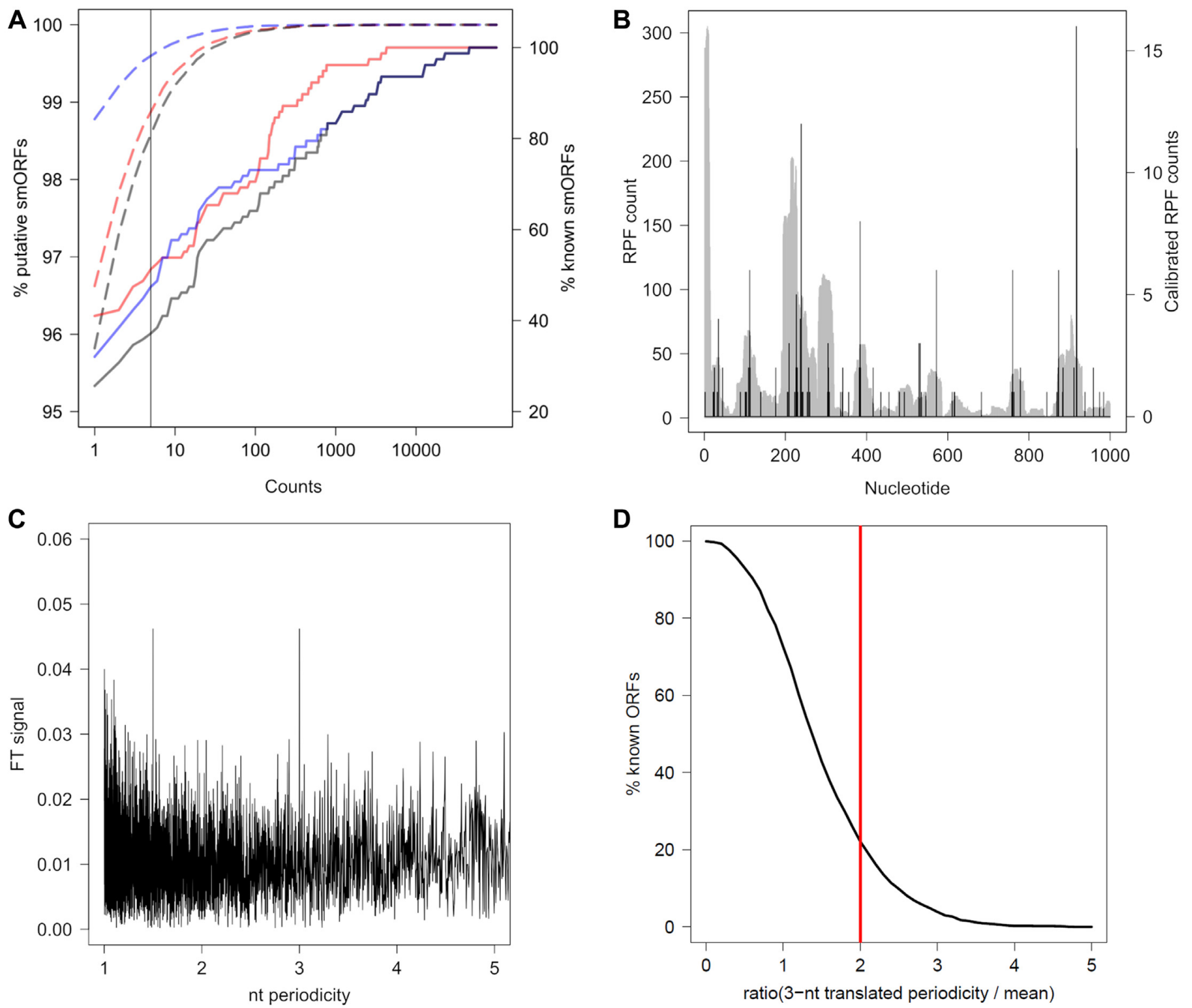

Figure 4. Detected smORFs for Ribo-Seq and TIS-Ribos-Seq data for E. coli and FT analysis of calibrated RPF counts. (A) Fraction of putative smORFs (dashed lines, left axis) and known smORFs (solid lines, right axis) (22) that are detected as translated and with genuine TIS with smORFer. Note the linear dependence of known smORFs that is caused by their different expression levels, while the putative smORF show a non-linear dependence. Red, translated (i.e. RPF counts); blue, with genuine TIS (i.e. RPF counts at TIS); black, both translated and with TIS counts. Vertical line denotes the cutoff $\geq 5$ RPFs. (B) RPF reads plotted in full length (grey, left axis) for the first $1000 \mathrm{nt}$ of the RNase I transcript compared to the calibrated RPF counts (black, right axis). (C) 3-nt periodicity FT signal of the calibrated RPFs for RNase I transcript. (D) Cumulative distributions of FT values for protein-coding ORFs. Vertical red line, cutoff of 2 .

Table 1. Comparison of the results for smORFs in three different organisms. For E. coli MG1655 Ribo-Seq and TIS-Ribo-Seq are available, whereas for B. subtilis and S. aureus only Ribo-Seq is available. Modules desigantion as in Figure 1

\begin{tabular}{|c|c|c|c|c|}
\hline Module & Description of the filtering step & E. coli & S. aureus & B. subtilis \\
\hline \multirow{2}{*}{$\begin{array}{l}\text { Genome-based smORF detection } \\
\text { (module A) }\end{array}$} & smORFs length $\geq 9$ to $\leq 150 \mathrm{nt}$ & 415133 & 305549 & 405321 \\
\hline & smORFs in non-annotated regions & 234399 & 179679 & 238446 \\
\hline \multirow{2}{*}{$\begin{array}{l}\text { Detection of translated ORFs } \\
\text { (module B) }\end{array}$} & translated smORFs with $\geq 5 \mathrm{RPF}$ & 3079 & 6595 & 2105 \\
\hline & $\begin{array}{l}\text { 3-nt translated smORFs with } 3 \text {-nt RPF } \\
\text { periodicity detected by Fourier transform }\end{array}$ & 175 & 555 & 168 \\
\hline \multirow{2}{*}{ Cross comparison } & translated smORFs and TIS-Ribo-Seq signal & 160 & n.a. & n.a. \\
\hline & $\begin{array}{l}\text { 3-nt translated smORFs with 3-nt RPF } \\
\text { periodicity and TIS-Ribo-Seq signal }\end{array}$ & 16 & n.a. & n.a. \\
\hline
\end{tabular}



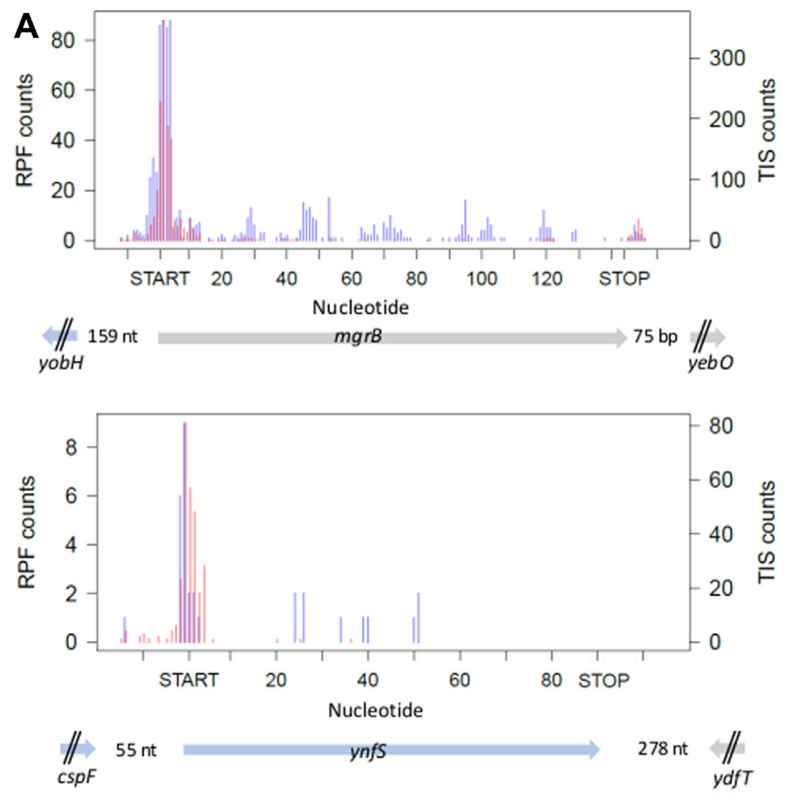
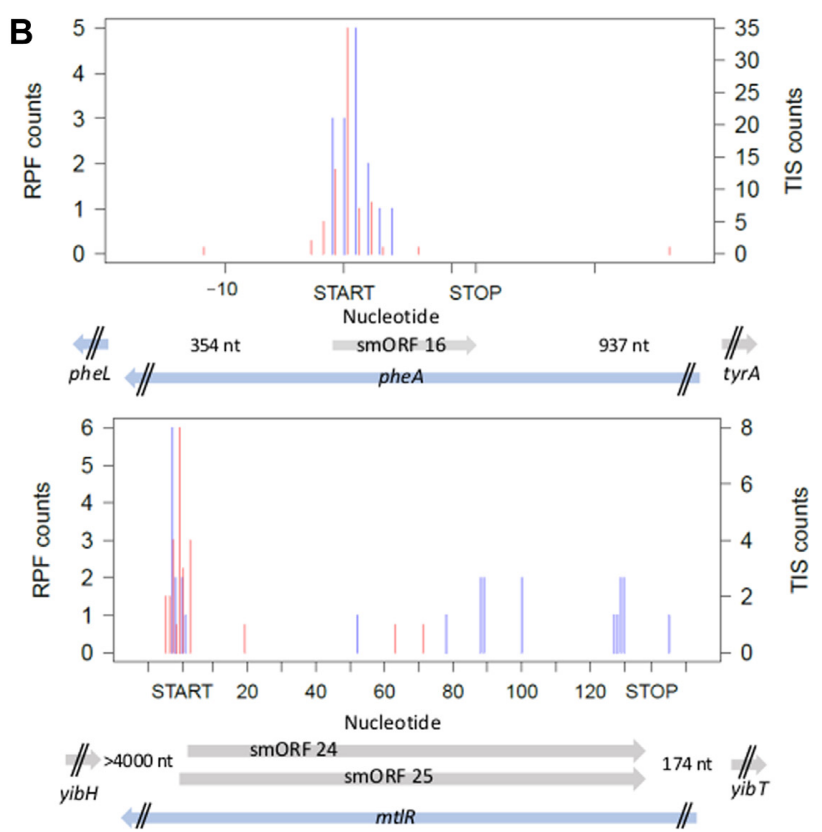

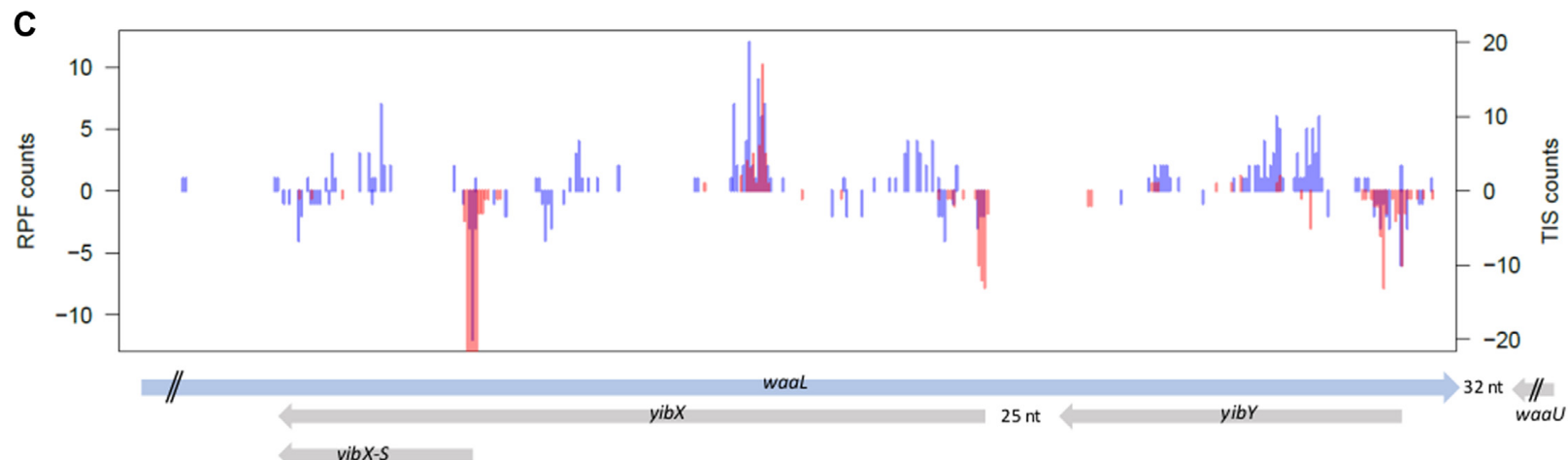

Figure 5. Examples of known and newly detected smORFs with smORFer in E. coli. (A) Examples of known and already experimentally verified smORFs (22) detected also with smORFer. (B) Examples of newly identified smORFs from each category, translated (upper panel) and 3nt-translated (lower panel). Lower panel: smORFer predicted two smORFs that differ only by their adjacent start codons. Since TIS-Seq counts are spread \pm one codon around the start codon (Supplementary Figure S2), there is no clear-cut indication for a preferred start. smORF 25 has two consecutive start codons (both TTG) and is by one start codon longer than smORF 24, otherwise both smORFs are identical. (C) Complex example of smORFs overlapping with known ORFs illustrating strand-specificity of RPF and TIS-Seq counts, and precise identification of smORF translational start site. All 3 smORFs, including also the short $y i b X$-S version of $y i b X$, are detected by smORFer and experimentally verified in (22). Counts displayed as positive values of the y-axes represent counts of ORFs located on the forward DNA strand, and negatively displayed counts of ORFs on the reverse strand. (A-C) Blue, RPF counts from the Ribo-Seq (left axis); red, counts from the TIS-Seq (right axis). ORFs architecture is shown at the bottom: blue arrow, ORFs located on the forward strand; gray, ORFs located on the reverse strand.; nt, denotes the distance to the next ORF; two black dashes, designate truncated, not-completely displayed adjacent ORFs.

In all three categories, i.e. the ORFs detected by the genome-based search, translated and 3-nt translated smORFs, which were verified in the E. coli TIS-Ribo-Seq data set (Table 1), we analyzed the distribution of the start codons. Among the 424 smORFs with TIS signal, the distribution of the smORFs initiated with ATG, GTG, TTG and CTG was-229, 65, 92 and 38, respectively. While for the 160 translated smORFs the distribution among the initiation codons was similar-79, 24, 43 and 14, respectively, for the 3-nt translated 16 candidates this changed to- -5 , 1, 7 and 3, respectively. This clearly distinct usage of start codons in smORFs, even within the most stringent - the 3-nt translated group, suggests that smORFs exhibit a dif- ferent bias of start codon usage than long protein-coding ORFs (49). Based on this distribution, it is conceivable to include a start-codon selection step in module A; however, TIS-Ribo-Seq data are available for only one organism and ideally this distribution, if uniform among prokaryotes as expected, should be experimentally verified for other bacteria.

For E. coli - the most studied organism-we sought to consider other datasets to further cross validate the predictions of smORFer. We used the most extensive mass spectrometry dataset available for the $E$. coli proteome (42). Using a Mascot score $>100$ and FDR $<0.01$ (46), from the total of 4245 protein-coding genes in E. coli, we detected 1890 
proteins $(\sim 44.5 \%)$. Applying the same criteria to the detected smORFs (1 unique peptide, 7 duplicates, best matching score 44), we detected only one candidate; 6 further candidates were selected with a score $<100$ (single peptides) (Supplementary Figure S5). The Ribo-Seq, TIS-Ribo-Seq and mass spectrometry show a good overlap in detecting known protein-coding genes (Supplementary Figure S6A), yet the depth of Ribo-Seq and even TIS-Ribo-Seq are much higher than mass spectrometry, that is likely the reason to limit the detection of smORFs. Most of the smORFs are expressed at much lower levels compared to long proteincoding ORFs (Supplementary Figure S6B). Furthermore, the trypsin-generated fragments are also non-unique, e.g. more than 2500 of the $3079 \mathrm{E}$. coli smORFs do not exhibit any unique peptide with $\geq 6$ amino acids (Supplementary Figure S6C).

We also considered recently published mass spectrometry data for $S$. aureus (41) and detected smORFs with SALT \& Pepper pipeline (https://gitlab.com/s.fuchs/pepper) (41). The pipeline uses a minimum of one unique peptide larger than 6 amino acids detected in at least two biological replicates and with a minimum score of 40 for unmodified and modified peptides, a minimum delta score of 6 for unmodified peptides and 17 for modified peptides, and a fixed false discovery rate (FDR) of 0.0001 for peptides and 0.01 for proteins (41). The $S A L T \&$ Pepper pipeline comprises genomic prediction of smORFs and mass spectrometry verification and detected in total 176 unique small proteins with a length of up to 100 amino acids. Thereby, 144 of them passed our criteria for genuinely translated, i.e. $\geq 5 \mathrm{PRF}$ as in the category for 3-nt translated smORFer candidates; 32 did not have any RPFs and were likely false-positives detected in SALT \& Pepper. Among the 144 candidates, 17 were with a length $\leq 50$ amino acids which is a selection criterion in smORFer.

Despite this fairly low overlap between the smORFs in the category $3 n$ t-translated (Table 1 ) and detected by mass spectrometry, it is of the same order of magnitude as observed for smORFs translated in Ribo-Seq data and detected by mass spectrometry for human cell lines (8). Besides the higher depth of the sequencing-based data than the mass spectrometry (62), several other reasons may contribute to the larger numbers of smORFs identified by deepsequencing approaches than by mass spectrometry (Supplementary Figure S6 and S7): (i) low expression level of smORFs, (ii) lack of unique peptides to be detected and uniquely assigned to smORFs, (iii) too short peptidasegenerated peptides to be detected by mass spectrometry, and (iv) conditional expression of smORFs under particular stress despite their constant translation in the translation noise. It should be also noted that mass spectrometry pipelines detect mainly soluble (small) proteins leaving out a large fraction of membrane or membrane-anchored proteins; the latter represent a significant fraction among small proteins $(5,38)$.

\section{Comparison of the performance of smORFer to other tools}

We compared smORFer to RibORF $(63,64)$ and GETORF (65). Similarly to smORFer, RibORF utilizes a multistep procedure, including Ribo-Seq data for detecting translated
Table 2. Comparison of the performance of smORFer with RibORF and GETORF in genome-based search for putative smORFs. The minimal and maximal length of smORFs was set at $9 \mathrm{nt}$ and $150 \mathrm{nt}$, respectively

\begin{tabular}{lccc}
\hline Algorithm & E. coli & S. aureus & B. subtilis \\
\hline GETORF & 180278 & 202597 & 200992 \\
RibORF & 415133 & 305549 & 405321 \\
smORFer & 415133 & 305549 & 405321 \\
unique stop codons & 176427 & 148408 & 179274 \\
RibORF/smORFer & & & \\
\hline
\end{tabular}

ORFs, similar minimal smORF length of $9 \mathrm{nt}$, thus, it is the most appropriate algorithm for comparison. GETORF is part of the EMBOSS suite and finds ORFs based on the nucleotide sequence and was chosen to compare the performance in detecting putative ORFs in the genomic module of smORFer (Figure 1). We performed three different comparisons: (i) general detection of putative smORFs from genomic sequences, (ii) detection of long ORFs which are usually well captured in various algorithms, and (iii) detection of smORFs in non-annotated regions. Notably, with the first criterion (i) smORFer and RibORF detected identical number of putative ORFs from the genomic sequences which was higher than these generated by GETORF (Table 2). Since both smORFer and RibORF detect ORFs with multiple starts but sharing the same stop codon, the number of detected ORFs is reduced when only stop codons were counted, i.e. considering one ORF per stop codon (Table 2).

The second comparison (ii) evaluates the results of RibORF and smORFer in detecting long ORFs, i.e. $>1000$ $\mathrm{nt}$, in the E. coli genome. Using genomic sequence, both RibORF and smORFer predicted a large number of putative ORFs, which however was much higher than the annotated ORFs in E. coli, as multiple start codons were considered which share the same stop codon (Table 3). Counting the ORFs by unique stop codon, $99.6 \%$ of the known annotated ORFs were detected by both algorithms. Including further criteria to select for translated ORFs, RibORF detected 235 translated ORFs $(1.2 \%$ of all known ORFs $>1000 \mathrm{nt}) \mathrm{com}-$ pared to $740(45 \%$ of all known ORFs $>1000 \mathrm{nt})$ detected by smORFer. In part, this is due to the utilization of TISRibo-Seq data, emphasizing the importance of using such data sets to precisely map initiation sites. It should be noted that RibORF, which does not use TIS-Ribo-Seq, runs much slower than our algorithm (2 days versus $6 \mathrm{~h}$ ).

Third (iii), we compared RibORF and smORFer by scanning only the non-annotated regions. RibORF detected 42, 463 and 1178 smORFs for E. coli, B. subtilis and S. aureus, respectively (Table 4). The number of validated smORF candidates by smORFer were higher 3079, 2105 and 6595 for E. coli, B. subtilis and S. aureus. Notably, for B. subtilis and $S$. aureus many smORFs predicted by RibORF overlapped with the set of translated candidates detected with smORFer (Table 4). The number of 3-nt translated smORFs was much lower and showed no overlap to the RibORF final candidates. While RibORF identified precisely larger ORFs (Table 3), smORFer outperformed it in detecting smORFs. This behavior is likely a result of the underlying assumption of RibORF which similarly to other algorithms identifies new ORFs using the same assumptions used for long ORFs 
Table 3. Comparison of the performance of smORFer and RibORF in identifying long ORFs (>1,000 nt) in E. coli

\begin{tabular}{|c|c|c|c|}
\hline RibORF results & & Overlap with known ORFs & Overlap with total known ORFs, $\%$ c \\
\hline ORFs in genome & $38284(1,811)^{\mathrm{a}}$ & $1576^{\mathrm{b}}$ & 99.6 \\
\hline 3-nt periodicity Ribo-Seq & $26850(1,185)$ & 1122 & 70.9 \\
\hline predicted ORFs & $235(235)$ & 19 & 1.2 \\
\hline ORFs in genome & $38284(1,811)^{\mathrm{a}}$ & $1576^{\mathrm{b}}$ & 99.6 \\
\hline translated $\mathrm{ORFs} \geq 5 \mathrm{RPFs}$ & $32949(1,483)$ & 1381 & 87.3 \\
\hline ORFs $\geq 5$ RPFs at start & $912(746)$ & 717 & 45.3 \\
\hline
\end{tabular}

${ }^{a}$ In brackets the ORFs counted by unique stop codons.

${ }^{\mathrm{b}}$ Some ORFs are not found by the genome search because they have non-canonical start or stop codons.

${ }^{\mathrm{c}}$ In total, there are 1582 annotated ORFs with length $>1000 \mathrm{nt}$ in $E$. coli.

Table 4. Comparison of the performance of smORFer and RibORF in detecting smORFs ( $\geq 9$ and $\leq 150 \mathrm{nt})$ within the non-annotated regions

\begin{tabular}{|c|c|c|c|c|}
\hline \multicolumn{2}{|c|}{ RibORF } & \multirow[t]{2}{*}{ Overlap } & \multicolumn{2}{|r|}{ smORFer } \\
\hline \multicolumn{4}{|c|}{ E. coli } & \\
\hline smORFs & 234399 & 234399 & 234399 & smORFs \\
\hline 3-nt periodicity & 546 & - & - & - \\
\hline \multirow[t]{5}{*}{ predicted ORFs } & 42 & 41 & 3079 & translated $\geq 5 \mathrm{RPFs}$ \\
\hline & & 0 & 175 & 3-nt translated \\
\hline & & 2 & 160 & translated ORFs and $\geq 5$ TIS counts at start \\
\hline & & 0 & 16 & 3-nt translated ORFs and $\geq 5$ TIS counts at start \\
\hline & & \multicolumn{3}{|r|}{ - } \\
\hline smORFs & 238446 & 238446 & 238446 & smORFs \\
\hline 3-nt periodicity & 1176 & - & - & - \\
\hline \multirow{3}{*}{ predicted ORFs } & 463 & 458 & 2105 & translated $\geq 5 \mathrm{RPFs}$ \\
\hline & & 0 & 168 & 3-nt translated \\
\hline & & \multicolumn{3}{|c|}{ S. aureus } \\
\hline smORFs & 179679 & 179679 & 179679 & smORFs \\
\hline 3-nt periodicity & 1821 & - & - & - \\
\hline predicted ORFs & 1178 & 1168 & 6595 & translated $\geq 5$ RPFs \\
\hline & & 0 & 555 & 3-nt translated ORFs \\
\hline
\end{tabular}

(66), namely $3 \mathrm{nt}$ periodicity as a diagnostic of a bona fide translation and protein conservation $(63,64)$. Many experimentally verified bacterial peptides encoded by smORFs exhibit different composition bias than the proteome encoded by long ORFs (67). In contrast, our results reveal characteristic features of smORFs that differ for long ORFs emphasizing on the importance of adjusting the selection criteria to the features of smORFs.

\section{CONCLUSION}

Comprehensively designed for annotating de novo smORFs using various data sets, smORFer presents remarkable advantages. It has a high efficiency in predicting smORFs with high probability to be expressed. The modularizable structure of smORFer offers advantages in verifying the smORFs calling dependent on the available data sets for each organism. The first part of module A, the genomebased ORF detection, is imperative as a starting point, since it sets the genomic boundaries of smORFs. The 3-nt sequence periodicity detection (FT, module $\mathrm{A}$ ) decreases the search space and we recommend using it when no further deep-sequencing data is available for the particular organism. For organisms, for which Ribo-Seq and/or TIS-Seq data are available, we recommend after the genomic search in module A to directly process with modules B and/or C. Both modules B and C can be applied independently depen- dent on the available deep-sequencing data sets: the higher the number of the data sets and the modules run in smORFer, the higher the accuracy of the smORF prediction. TISRibo-Seq is particularly powerful in unambiguously assigning overlapping smORFs.

Deep-sequencing-based approaches offer higher depth than mass spectrometry. However, to decrease the number of false-positives, several approaches should be combined (i.e. Ribo-Seq combined with TIS-Ribo-Seq to select for genuine initiation, and/or with Term-Seq for determining faithful termination (68)). Sequencing approaches delineating initiation and termination are in particular useful in genomes with overlapping reading-frames architecture as the prokaryotic genomes. smORFer is also suitable for eukaryotes; we recommend running module $\mathrm{A}$ on the transcriptome since eukaryotic genomes can reveal extremely large number of smORFs requiring a large computation power.

Many smORFs might be expressed only under stress conditions. Hence, the next challenge is to surgically dissect their expression with Ribo-Seq and TIS-Ribo-Seq collected under various stress conditions. This will allow conditionally translated smORFs to be disambiguated from the pool of smORFs with no RPFs under permissive conditions, i.e. categorized as untranslated. When paired to smORFer such data sets, expression events, even conditional expression events, will be mapped more comprehensively. 
Computationally, smORFer enables full analysis in a standardized way requiring little computational resources. The workflow in each module is easy to use and simple to modify to achieve high precision in smORFs calling.

\section{DATA AVAILABILITY}

Raw sequencing data including the RPM tables have been deposited within Gene Expression Omnibus (GEO) under accession number GSE150601.

All scripts used for the calibration of RPFs are available at https:/github.com/AlexanderBartholomaeus/ MiMB_ribosome_profiling. Scripts, example calls and files for smORFer using $E$. coli data sets are available at https: //github.com/AlexanderBartholomaeus/smORFer.

\section{SUPPLEMENTARY DATA}

Supplementary Data are available at NAR Online.

\section{FUNDING}

Deutsche Forschungsgemeinschaft SPP 2002 [IG 73/16-1 to Z.I. and GRK PROCOMPAS to S.E.]. Funding for open access charge: DFG.

Conflict of interest statement. None declared.

\section{REFERENCES}

1. Fickett,J.W. (1982) Recognition of protein coding regions in DNA sequences. Nucleic Acids Res., 10, 5303-5318.

2. Basrai,M.A., Hieter,P. and Boeke,J.D. (1997) Small open reading frames: beautiful needles in the haystack. Genome Res. , 7, 768-771.

3. Maeda,N., Kasukawa,T., Oyama,R., Gough,J., Frith,M., Engstrom,P.G., Lenhard,B., Aturaliya,R.N., Batalov,S., Beisel,K.W. et al. (2006) Transcript annotation in FANTOM3: mouse gene catalog based on physical cDNAs. PLos Genet., 2, e62.

4. Angiuoli,S.V., Gussman,A., Klimke,W., Cochrane,G., Field,D., Garrity,G., Kodira,C.D., Kyrpides,N., Madupu,R., Markowitz,V. et al. (2008) Toward an online repository of Standard Operating Procedures (SOPs) for (meta)genomic annotation. OMICS, 12, 137-141.

5. Ramamurthi,K.S. and Storz,G. (2014) The small protein floodgates are opening; now the functional analysis begins. BMC Biol., 12, 96.

6. Andrews,S.J. and Rothnagel,J.A. (2014) Emerging evidence for functional peptides encoded by short open reading frames. Nat. Rev. Genet., 15, 193-204.

7. Anderson,D.M., Anderson,K.M., Chang,C.L., Makarewich,C.A., Nelson,B.R., McAnally,J.R., Kasaragod,P., Shelton,J.M., Liou,J., Bassel-Duby,R. et al. (2015) A micropeptide encoded by a putative long noncoding RNA regulates muscle performance. Cell, $\mathbf{1 6 0}$, 595-606.

8. Chen,J., Brunner,A.D., Cogan,J.Z., Nunez,J.K., Fields,A.P., Adamson,B., Itzhak,D.N., Li,J.Y., Mann,M., Leonetti,M.D. et al. (2020) Pervasive functional translation of noncanonical human open reading frames. Science, 367, 1140-1146.

9. D'Lima,N.G., Ma,J., Winkler,L., Chu,Q., Loh,K.H., Corpuz,E.O., Budnik,B.A., Lykke-Andersen,J., Saghatelian,A. and Slavoff,S.A (2017) A human microprotein that interacts with the mRNA decapping complex. Nat. Chem. Biol., 13, 174-180.

10. Jackson,R., Kroehling,L., Khitun,A., Bailis,W., Jarret,A., York,A.G., Khan,O.M., Brewer,J.R., Skadow,M.H., Duizer,C. et al. (2018) The translation of non-canonical open reading frames controls mucosal immunity. Nature, 564, 434-438.

11. Kondo,T., Plaza,S., Zanet,J., Benrabah,E., Valenti,P., Hashimoto,Y., Kobayashi,S., Payre,F. and Kageyama,Y. (2010) Small peptides switch the transcriptional activity of Shavenbaby during Drosophila embryogenesis. Science, 329, 336-339.
12. Matsumoto,A., Pasut,A., Matsumoto,M., Yamashita,R., Fung,J., Monteleone,E., Saghatelian,A., Nakayama,K.I., Clohessy,J.G. and Pandolfi,P.P. (2017) mTORC1 and muscle regeneration are regulated by the LINC00961-encoded SPAR polypeptide. Nature, 541, 228-232.

13. Nelson,B.R., Makarewich,C.A., Anderson,D.M., Winders,B.R., Troupes,C.D., Wu,F., Reese,A.L., McAnally,J.R., Chen,X., Kavalali,E.T. et al. (2016) A peptide encoded by a transcript annotated as long noncoding RNA enhances SERCA activity in muscle. Science, 351, 271-275.

14. Araujo-Bazan,L., Ruiz-Avila,L.B., Andreu,D., Huecas,S. and Andreu,J.M. (2016) Cytological Profile of Antibacterial FtsZ Inhibitors and Synthetic Peptide MciZ. Front Microbiol, 7, 1558.

15. Bobrovskyy,M. and Vanderpool,C.K. (2014) The small RNA SgrS: roles in metabolism and pathogenesis of enteric bacteria. Front. Cell Infect. Microbiol., 4, 61 .

16. Ebmeier,S.E., Tan,I.S., Clapham,K.R. and Ramamurthi,K.S. (2012) Small proteins link coat and cortex assembly during sporulation in Bacillus subtilis. Mol. Microbiol., 84, 682-696.

17. Hobbs,E.C., Yin,X., Paul,B.J., Astarita,J.L. and Storz,G. (2012) Conserved small protein associates with the multidrug efflux pump AcrB and differentially affects antibiotic resistance. Proc. Natl. Acad. Sci. U.S.A., 109, 16696-16701.

18. Hobbs,E.C., Fontaine,F., Yin,X. and Storz,G. (2011) An expanding universe of small proteins. Curr. Opin. Microbiol., 14, 167-173.

19. Meydan,S., Marks,J., Klepacki,D., Sharma,V., Baranov,P.V., Firth,A.E., Margus,T., Kefi,A., Vazquez-Laslop,N. and Mankin,A.S (2019) Retapamulin-assisted ribosome profiling reveals the alternative Bacterial proteome. Mol. Cell, 74, 481-493.

20. Modell,J.W., Kambara,T.K., Perchuk,B.S. and Laub,M.T. (2014) A DNA damage-induced, SOS-independent checkpoint regulates cell division in Caulobacter crescentus. PLoS Biol., 12, e1001977.

21. Salazar,M.E., Podgornaia,A.I. and Laub,M.T. (2016) The small membrane protein MgrB regulates PhoQ bifunctionality to control PhoP target gene expression dynamics. Mol. Microbiol., 102, 430-445.

22. Weaver,J., Mohammad,F., Buskirk,A.R. and Storz,G. (2019) Identifying small proteins by ribosome profiling with stalled initiation complexes. mBio, 10, e02819-18.

23. Ingolia,N.T., Ghaemmaghami,S., Newman,J.R. and Weissman,J.S. (2009) Genome-wide analysis in vivo of translation with nucleotide resolution using ribosome profiling. Science, 324, 218-223.

24. Chun,S.Y., Rodriguez,C.M., Todd,P.K. and Mills,R.E. (2016) SPECtre: a spectral coherence-based classifier of actively translated transcripts from ribosome profiling sequence data. $B M C$ Bioinformatics, 17, 482.

25. Ingolia,N.T., Lareau,L.F. and Weissman,J.S. (2011) Ribosome profiling of mouse embryonic stem cells reveals the complexity and dynamics of mammalian proteomes. Cell, 147, 789-802.

26. Xiao,Z., Huang,R., Xing,X., Chen,Y., Deng,H. and Yang,X. (2018) De novo annotation and characterization of the translatome with ribosome profiling data. Nucleic Acids Res., 46, e61.

27. Guttman,M., Russell,P., Ingolia,N.T., Weissman,J.S. and Lander,E.S. (2013) Ribosome profiling provides evidence that large noncoding RNAs do not encode proteins. Cell, 154, 240-251.

28. Aspden,J.L., Eyre-Walker,Y.C., Phillips,R.J., Amin,U., Mumtaz,M.A., Brocard,M. and Couso,J.P. (2014) Extensive translation of small Open Reading Frames revealed by Poly-Ribo-Seq. Elife, 3, e03528.

29. Heyer,E.E. and Moore,M.J. (2016) Redefining the Translational Status of 80S Monosomes. Cell, 164, 757-769.

30. Biever,A., Glock,C., Tushev,G., Ciirdaeva,E., Dalmay,T., Langer,J.D. and Schuman,E.M. (2020) Monosomes actively translate synaptic mRNAs in neuronal processes. Science, 367, eaay4991.

31. Fields,A.P., Rodriguez,E.H., Jovanovic,M., Stern-Ginossar,N., Haas,B.J., Mertins,P., Raychowdhury,R., Hacohen,N., Carr,S.A., Ingolia,N.T. et al. (2015) A Regression-based analysis of ribosome-profiling data reveals a conserved complexity to mammalian translation. Mol. Cell, 60, 816-827.

32. Hsu,P.Y., Calviello,L., Wu,H.L., Li,F.W., Rothfels,C.J., Ohler,U. and Benfey,P.N. (2016) Super-resolution ribosome profiling reveals unannotated translation events in Arabidopsis. Proc. Natl. Acad. Sci. U.S.A., 113, E7126-E7135.

33. Nakahigashi,K., Takai,Y., Kimura,M., Abe,N., Nakayashiki,T., Shiwa,Y., Yoshikawa,H., Wanner,B.L., Ishihama,Y. and Mori,H. 
(2016) Comprehensive identification of translation start sites by tetracycline-inhibited ribosome profiling. DNA Res., 23, 193-201.

34. Stern-Ginossar,N., Weisburd,B., Michalski,A., Le,V.T., Hein,M.Y., Huang,S.X., Ma,M., Shen,B., Qian,S.B., Hengel,H. et al. (2012) Decoding human cytomegalovirus. Science, 338, 1088-1093.

35. Bazzini,A.A., Johnstone,T.G., Christiano,R., Mackowiak,S.D., Obermayer,B., Fleming,E.S., Vejnar,C.E., Lee,M.T., Rajewsky,N., Walther,T.C. et al. (2014) Identification of small ORFs in vertebrates using ribosome footprinting and evolutionary conservation. EMBO J., 33, 981-993.

36. Calviello,L., Mukherjee,N., Wyler,E., Zauber,H., Hirsekorn,A., Selbach,M., Landthaler,M., Obermayer,B. and Ohler,U. (2016) Detecting actively translated open reading frames in ribosome profiling data. Nat. Methods, 13, 165-170.

37. Shell,S.S., Wang,J., Lapierre,P., Mir,M., Chase,M.R., Pyle,M.M., Gawande,R., Ahmad,R., Sarracino,D.A., Ioerger,T.R. et al. (2015) Leaderless transcripts and small proteins are common features of the mycobacterial translational landscape. PLos Genet., 11, e1005641.

38. Storz,G., Wolf,Y.I. and Ramamurthi,K.S. (2014) Small proteins can no longer be ignored. Annu. Rev. Biochem., 83, 753-777.

39. Eastman,G., Smircich,P. and Sotelo-Silveira,J.R. (2018) Following ribosome footprints to understand translation at a genome wide level. Comput Struct Biotechnol J, 16, 167-176.

40. Li,G.W., Oh,E. and Weissman,J.S. (2012) The anti-Shine-Dalgarno sequence drives translational pausing and codon choice in bacteria. Nature, 484, 538-541.

41. Fuchs,S., Kucklick,M., Lehmann,E., Beckann,A., Wilkens,M., Kolte,B., Mustafayeva,A., Ludwig,T., Diwo,M., Wissing,J. et al. (2021)Towards the characterization of the hidden world of small proteins in Staphylococcus aureus, a proteogenomics approach. Plos Genet., 17, e1009585.

42. Schmidt,A., Kochanowski,K., Vedelaar,S., Ahrne,E., Volkmer,B., Callipo,L., Knoops,K., Bauer,M., Aebersold,R. and Heinemann,M. (2016) The quantitative and condition-dependent Escherichia coli proteome. Nat. Biotechnol., 34, 104-110.

43. Del Campo,C., Bartholomaus,A., Fedyunin,I. and Ignatova,Z. (2015) Secondary structure across the bacterial transcriptome reveals versatile roles in mRNA regulation and function. PLoS Genet., 11, e1005613.

44. Langmead,B., Trapnell,C., Pop,M. and Salzberg,S.L. (2009) Ultrafast and memory-efficient alignment of short DNA sequences to the human genome. Genome Biol., 10, R25.

45. Perez-Riverol,Y., Csordas,A., Bai,J., Bernal-Llinares,M., Hewapathirana,S., Kundu,D.J., Inuganti,A., Griss,J., Mayer,G., Eisenacher,M. et al. (2019) The PRIDE database and related tools and resources in 2019: improving support for quantification data. Nucleic Acids Res., 47, D442-D450.

46. Perkins,D.N., Pappin,D.J., Creasy,D.M. and Cottrell,J.S. (1999) Probability-based protein identification by searching sequence databases using mass spectrometry data. Electrophoresis, 20, 3551-3567.

47. Quinlan,A.R. and Hall,I.M. (2010) BEDTools: a flexible suite of utilities for comparing genomic features. Bioinformatics, 26, 841-842.

48. Baek,J., Lee,J., Yoon,K. and Lee,H. (2017) Identification of unannotated small genes in Salmonella. G3 (Bethesda), 7, 983-989.

49. Hecht,A., Glasgow,J., Jaschke,P.R., Bawazer,L.A., Munson,M.S., Cochran,J.R., Endy,D. and Salit,M. (2017) Measurements of translation initiation from all 64 codons in E. coli. Nucleic Acids Res., 45, 3615-3626.
50. Del Campo,C. and Ignatova,Z. (2016) Probing dimensionality beyond the linear sequence of mRNA. Curr. Genet., 62, 331-334.

51. Bartholomaus,A. and Ignatova,Z. (2021) Codon resolution analysis of ribosome profiling data. Methods Mol. Biol., 2252, 251-268.

52. Woolstenhulme,C.J., Guydosh,N.R., Green,R. and Buskirk,A.R. (2015) High-precision analysis of translational pausing by ribosome profiling in bacteria lacking EFP. Cell Rep., 11, 13-21.

53. Bartholomaus,A., Del Campo,C. and Ignatova,Z. (2016) Mapping the non-standardized biases of ribosome profiling. Biol. Chem., 397, $23-35$.

54. Dunn,J.G. and Weissman,J.S. (2016) Plastid: nucleotide-resolution analysis of next-generation sequencing and genomics data. $B M C$ Genomics, 17, 958.

55. Popa,A., Lebrigand,K., Paquet,A., Nottet,N., Robbe-Sermesant,K., Waldmann,R. and Barbry,P. (2016) RiboProfiling: a Bioconductor package for standard Ribo-seq pipeline processing. F1000Res, $\mathbf{5}$, 1309.

56. Lauria,F., Tebaldi,T., Bernabo,P., Groen,E.J.N., Gillingwater,T.H and Viero,G. (2018) riboWaltz: optimization of ribosome P-site positioning in ribosome profiling data. PLoS Comput. Biol., 14, e1006169.

57. Davidovich,C., Bashan,A., Auerbach-Nevo,T., Yaggie,R.D., Gontarek,R.R. and Yonath,A. (2007) Induced-fit tightens pleuromutilins binding to ribosomes and remote interactions enable their selectivity. Proc. Natl Acad. Sci. U.S. A., 104, 4291-4296.

58. Yan,K., Madden,L., Choudhry,A.E., Voigt,C.S., Copeland,R.A. and Gontarek,R.R. (2006) Biochemical characterization of the interactions of the novel pleuromutilin derivative retapamulin with bacterial ribosomes. Antimicrob. Agents Chemother, 50, 3875-3881.

59. Orr,M.W., Mao,Y., Storz,G. and Qian,S.B. (2020) Alternative ORFs and small ORFs: shedding light on the dark proteome. Nucleic Acids Res., 48, 1029-1042.

60. Shabalina,S.A., Ogurtsov,A.Y. and Spiridonov,N.A. (2006) A periodic pattern of mRNA secondary structure created by the genetic code. Nucleic Acids Res., 34, 2428-2437.

61. Tiwari,S., Ramachandran,S., Bhattacharya,A., Bhattacharya,S. and Ramaswamy,R. (1997) Prediction of probable genes by Fourier analysis of genomic sequences. Comput. Appl. Biosci., 13, 263-270.

62. Schwanhausser,B., Busse,D., Li,N., Dittmar,G., Schuchhardt,J., Wolf,J., Chen,W. and Selbach,M. (2011) Global quantification of mammalian gene expression control. Nature, 473, 337-342.

63. Ji,Z. (2018) RibORF: Identifying genome-wide translated open reading frames using ribosome profiling. Curr. Protoc. Mol. Biol., 124, e67.

64. Ji,Z., Song,R., Regev,A. and Struhl,K. (2015) Many lncRNAs, $5^{\prime}$ UTRs, and pseudogenes are translated and some are likely to express functional proteins. Elife, 4, e08890.

65. Rice,P., Longden,I. and Bleasby,A. (2000) EMBOSS: the European Molecular Biology Open Software Suite. Trends Genet., 16, 276-277.

66. Ndah,E., Jonckheere,V., Giess,A., Valen,E., Menschaert,G. and Van Damme,P. (2017) REPARATION: ribosome profiling assisted (re-)annotation of bacterial genomes. Nucleic Acids Res., 45, e168.

67. Hemm,M.R., Paul,B.J., Schneider,T.D., Storz,G. and Rudd,K.E. (2008) Small membrane proteins found by comparative genomics and ribosome binding site models. Mol. Microbiol., 70, 1487-1501.

68. Adams,P.P., Baniulyte,G., Esnault,C., Chegireddy,K., Singh,N., Monge,M., Dale,R.K., Storz,G. and Wade,J.T. (2021) Regulatory roles of Escherichia coli $5^{\prime}$ UTR and ORF-internal RNAs detected by $3^{\prime}$ end mapping. Elife, 10, e62438. 